\title{
Hacia un Modelo de Comunicación Comunitaria para el Pos-acuerdo. Caso: región del Ariari, Colombia
}

Towards a community communication model for the post-agreement. Case: Ariari region, Colombia

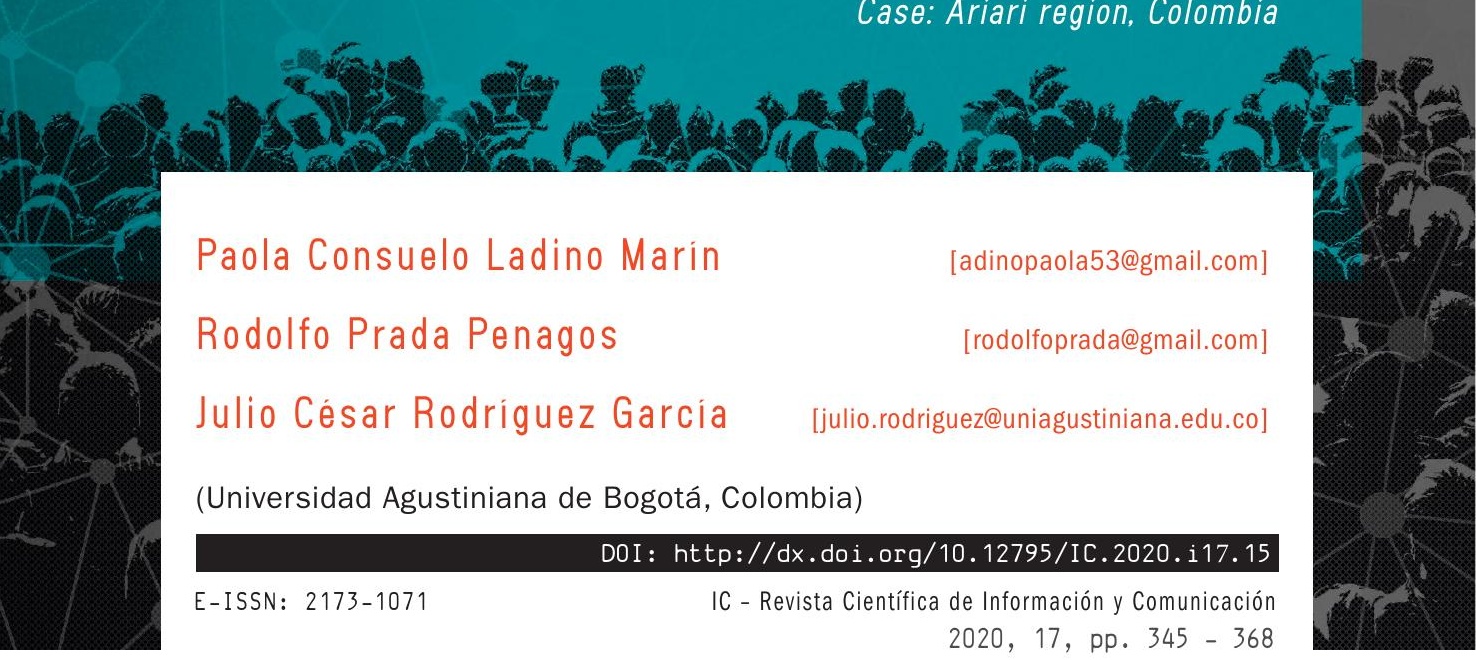

\section{Resumen}

Este artículo propone un modelo de comunicación comunitaria para la región colombiana del Ariari, en un contexto particularmente impactado por el Acuerdo de Paz suscrito entre el gobierno colombiano y el grupo guerrillero Farc-EP. La investigación se enfocó en los medios de comunicación comunitarios que operan en la región, bajo la premisa de que estos emergen como actores fundamentales en la construcción de un escenario de posconflicto, en una zona históricamente afectada por diversas manifestaciones de violencia (Zúñiga \& Grattan, 2017). La investigación, de carácter cualitativo, permite plantear una propuesta que articule los medios comunitarios en los ámbitos de los enunciadores, las fuentes de información, el mensaje y los receptores, para responder al reto de transformar la sociedad regional hacia la búsqueda del desarrollo humano sostenible y deseable.

\section{Abstract}

This article proposes a model of community communication for the Colombian region of Ariari, in a context particularly impacted by the Peace Agreement signed between the Colombian government and the Farc-EP guerrilla group. The investigation focused on community media that operate in the 
region, under the premise that they emerge as fundamental actors in the construction of a postconflict scenario, in an area historically affected by various manifestations of violence (Zúñiga \& Grattan, 2017). The qualitative research allows us to propose a proposal that articulates community media in the fields of enunciators, sources of information, message and recipients, to respond to the challenge of transforming regional society towards the search for sustainable human development and desirable.

\section{Palabras clave}

Paz, Medios comunitarios, Ariari, Modelo de comunicación.

\section{Keywords}

Peace, Community media, Ariari, Communication model.

\section{Sumario}

1. Introducción

2. El concepto de modelo como una representación gráfica de la realidad

3. Antecedentes de un modelo de comunicación para la paz

4. Metodología

5. Caracterización de los medios comunitarios de Ariari

6. Resultador: Gestión de los medios y factores que proponen para completar el modelo de comunicación comunitaria

7. Discusión y conclusiones: Modelo de comunicación comunitaria

7.1. Ejes del modelo de comunicación comunitario

\section{Summary}

1. Introduction

2. The concept of model as a graphic representation of reality

3. Background of a communication model for peace

4. Methodology

5. Characterization of the community media of the Ariari

6. Results: Management of the means and factors that are proposed to complement the community communication model

7. Discussion and conclusions: Community communication model

7.1 Axes of the community communication model 


\section{Introducción}

El Ariari es una de las regiones de Colombia con mayor riqueza agraria y ganadera, considerada la despensa agrícola del país, con variedad climática, potencial turístico y diversidad hídrica (Instituto Departamental de Cultura del Meta, 2016). Es un terreno tan amplio que se ha convertido en el epicentro de diferentes intereses políticos, económicos y geoestratégicos. De acuerdo con Alarcón, Huffington, Mosquera, \& Bermúdez (2017), las comunidades campesinas han buscado impulsar planes agrícolas que optimicen la productividad; los latifundistas, movilizar la ganadería extensiva; la guerrilla, desplegar una riqueza ilegal y establecerse en el territorio; los paramilitares, mantener control espacial y suscitar la ilegalidad; las multinacionales, estimular el sector minero-energético; y el Estado, implantar un esquema neoliberal economicista, que ha generado tensiones y conflictos que han llevado a la desigualdad y el conflicto social.

En este orden de ideas, y bajo un contexto tan complejo y diverso, los medios comunitarios toman un valor especial, cuando en zonas de conflicto podrían emerger para fortalecer la democracia e incentivar la participación ciudadana. Cuando trabajan en medio de la comunidad, no solo para informar, sino transformar, estos medios pueden educar, sensibilizar social y ambientalmente, promover dinámicas económicas locales, conservar la tradición cultural y la idiosincrasia, destacar el respeto por la igualdad y los Derechos Humanos, inculcar valores, en últimas, conservar la identidad. Según Jesús Martín Barbero, citado por (Reguero, 2008, pág. 1), "Ia identidad no es un hecho, sino un relato. Las culturas permanecen vivas mientras se comunican entre ellas, siendo la comunicación una dimensión constitutiva de la vida cultural y no sólo su exhibición".

De esta manera, los medios comunitarios ${ }^{1}$ se convierten en espacios que convocan el interés público y que responden a dinámicas distintas a las de los medios masivos comerciales, especialmente en lo que concierne con la visibilidad de las problemáticas locales. Corresponde a los medios comunitarios una labor en la que se reconozca al otro y se marque distancia de las lógicas de los medios masivos que suelen

1 En el contexto mediático colombiano, a los medios comunitarios se les suele señalar también como "medios alternativos". En esta investigación se acudirá solamente al concepto de "comunitario", claramente definido en la legislación que regula el funcionamiento de este tipo de medios. 
convertirse en eco de los estamentos de poder. En este orden de ideas, conviene recordar lo afirmado por Jesús Barbero, citado por Reguero (2008):

Lo público ha sido fagocitado por lo estatal provocando la decadencia de lo social y de su heterogeneidad. Ante medios de comunicación ventrílocuos necesitamos políticas que permitan la expresión de otras voces. El desafío fundamental de las políticas públicas es plantearse la heterogeneidad de lo social (p.1).

Se espera de los medios comunitarios la elaboración de un discurso alternativo al discurso dominante de los medios masivos en el que no se visibiliza a la región y, por el contrario, esta se relega a espacios marginales en la agenda informativa.

En Colombia, los medios comunitarios potenciaron su actuación luego de un compromiso que el gobierno adquirió en la Cumbre Mundial sobre la Sociedad de la Información (Ginebra, 2003) y (Túnez, 2005) y con la Convención Sobre la Protección y Promoción de la Diversidad de las Expresiones Culturales (Zúñiga \& Grattan, 2017).

En este contexto, resulta pertinente proponer un modelo de comunicación comunitaria sobre la base de repensar las esferas de la sociedad regional y local, y de identificar las necesidades de las comunidades en la búsqueda de un escenario de desarrollo social y convivencia ciudadana. En últimas, un modelo que integre acciones y actores de los medios comunitarios para dinamizar su impacto social.

\section{El concepto de modelo como una representación gráfica de la realidad}

Bajo la idea de modelo se han definido diversas concepciones. Para Caracheo (2002), el modelo se relaciona con la explicación de un fenómeno para establecer un canon o guía de acción, un prototipo o serie de objetos similares. Los modelos son una descripción gráfica de una parte de la realidad que muestra las principales partes de un proceso y su interrelación. Se presentan como una mirada amplia que ayuda a descifrar y simplificar información, sirviendo de guía para quien interpreta los aspectos clave del proceso, generando una predicción exitosa del curso de ciertos eventos Castro y Moreno (2006).

Sesento (2008) describe los modelos como "construcciones mentales que permiten una aproximación a la realidad de un fenómeno, distinguiendo sus características 
para facilitar su comprensión" (p.12). Por su parte, Villanueva, Barranquero y Ramos (2016) afirman que el modelo se comprende como la representación de un sistema desarrollado con un propósito específico. De manera que el término modelo se vincula con la representación de un hecho, situación o fenómeno como ideal a seguir, ya que busca visibilizar características generales desde su estructura, para explicar procesos, mecanismos o factores que se interrelacionan y que están sustentados teóricamente para facilitar su comprensión.

En el propósito de proponer un modelo de comunicación, este debe vincular diversos ejes y remitir a distintos contextos sociales que involucran un sinnúmero de relaciones. Centrarse en la noción comunicativa implica considerar estructuras de producción de sentido.

\section{Antecedentes de un modelo de comunicación para la paz}

Para esta investigación se tomó como referente conceptual el modelo de Comunicación para el Desarrollo y la Paz planteado por Toro (2014), quien señala que el propósito del modelo es "contribuir a la transformación de una realidad violenta presente, concebida no solo desde el conflicto armado, también desde la violencia estructural, directa y cultural, hacia una realidad pacífica futura".

Según la autora, la comunicación para el desarrollo y la paz es un eje estructural y transversal de los procesos orientados a la promoción y consecución del desarrollo humano integral y sostenible, porque acerca a la gente y la pone a dialogar, a priorizar y a decidir. Como tal, se constituye en un componente intrínseco de los planes, programas y proyectos de desarrollo en el territorio de interés.

Como lo ilustra la figura número 1 , en el modelo de Toro los elementos centrales que le siguen al núcleo fundamental del desarrollo son: 1) la participación crítica y consciente, 2) las redes -colectivos, medios de comunicación y redes sociales-, 3) la movilización y el cambio social y 4) la cultura de paz. Estos elementos facilitan la construcción del desarrollo en un Estado social de derecho, cuyos principios se afincan en la democracia, la libertad, la igualdad, la justicia y la solidaridad (Pág., 11). 


\section{FIGURA 1 \\ Modelo de Comunicación para el Desarrollo y la Paz en el Oriente Antioqueño, Colombia}

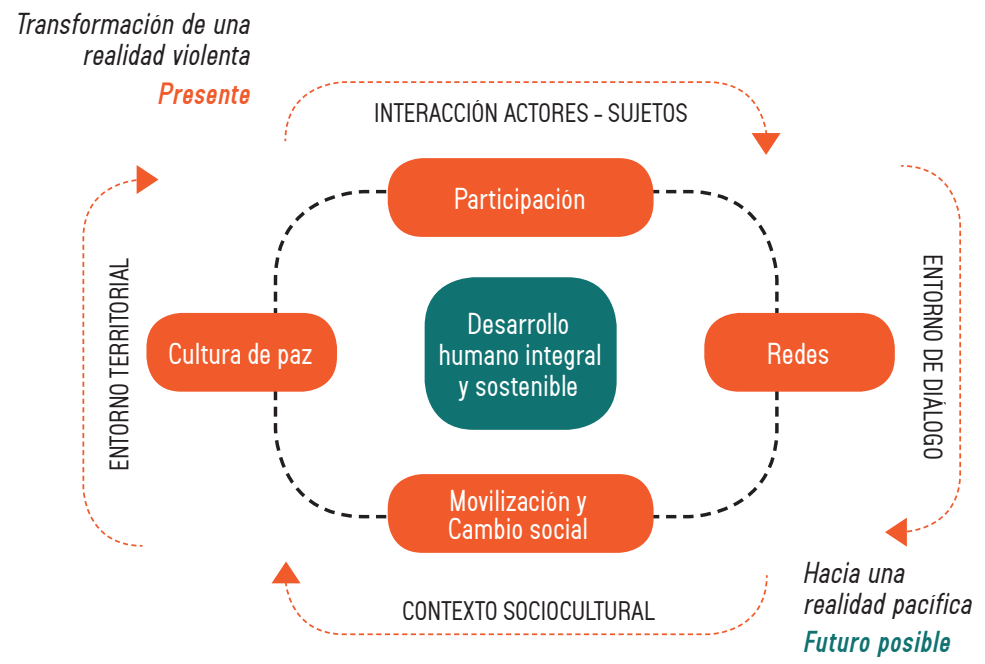

Fuente: Toro, 2014.

Desde esta perspectiva, plantear un modelo cuyo núcleo sean los medios comunitarios en una zona de conflicto territorial implica visibilizar los procesos comunitarios que aporten a una cultura de paz, los cuales, al ser modelados, susciten otras formas de comunicación válidas en el desarrollo de comunidades para la paz.

Este proceso conlleva un ejercicio hermenéutico y de análisis que permita tener una visión holística de las condiciones que emergen del acuerdo de paz suscrito entre el gobierno colombiano y las Farc-EP, mediante el cual se cerró un contexto de conflicto armado (Toro 2014). La región del Ariari ha padecido desplazamiento forzado, secuestro, extorsión, producción de cultivos "ilícitos", violencia armada y violaciones a los derechos humanos que, luego de la firma del Acuerdo General para la Terminación del Conflicto y la Construcción de una Paz Estable y Duradera" (Oficina del Alto Comisionado para la Paz, $\mathrm{s} / \mathrm{f}$ ), han bajado sensiblemente en intensidad y alimentado la esperanza de un desarrollo social basado en la convivencia y la reconciliación.

En este orden de ideas, esta investigación sugiere un modelo de comunicación soportado en los medios de comunicación comunitarios, en el entendido de que estos 
actúan como "mediadores entre la realidad y la comunidad, porque amplían el acceso a nuevas oportunidades y generan diálogo entre la comunidad y los entes encargados en la toma de decisiones" (Oficina de Políticas para el Desarrollo, 2011).

\section{Metodología}

Esta investigación, cuyo propósito es la formulación de un modelo de comunicación comunitaria para la región del Ariari, se trabajó desde un enfoque cualitativo. De acuerdo con Rueda (2007), en la investigación cualitativa intervienen diversas visiones que buscan identificar fenómenos o determinar relaciones que se dan dentro de los componentes analizados.

En este sentido, se plantearon varias fases. En un primer momento, se realizó una revisión documental acerca de la región del Ariari para comprender las lógicas comunicacionales a las que responden los medios comunitarios de la región. En un segundo momento, se realizó una caracterización de los medios de comunicación comunitaria a partir de un instrumento que permitió identificar tipos de medios, públicos objetivos, tendencias de contenido, coberturas y periodicidad, antigüedad, modelos de financiación y oportunidades de mejoramiento.

Se tomó como antecedente la Cartografía de Medios Comunitarios en el Meta elaborada por la Fundación para la Libertad de Prensa (2015), en la cual se observa que en ese departamento existen 50 medios comunitarios, 14 de los cuales operan en las tres subregiones del Ariari (ver tabla 1).

TABLA 1

\section{Medios de comunicación comunitarios y/o alternativos en el Ariari}

Municipio

Alto Ariari
Habitantes

Nümero Medios

Canal

\begin{tabular}{|c|c|c|c|}
\hline El castillo & 6127 & 1 & Radio \\
\hline Cubarral & 6261 & 1 & Radio \\
\hline El Dorado & 3457 & 0 & No tiene
\end{tabular}




\begin{tabular}{c|c|c|c|}
\hline Municipio & Habitantes & Número Medios & Canal \\
\hline San Martin & 25902 & 0 & No tiene \\
\hline Lejanías & 9273 & 0 & No tiene \\
\hline
\end{tabular}

\section{Medio Ariari}

\begin{tabular}{|c|c|c|c|}
\hline Fuente de Oro & 14139 & 1 & Tv comercial \\
\hline San juan de Arama & 8692 & 1 & Radio \\
\hline Granada & 67192 & 7 & Radio, Prensa TV \\
\hline
\end{tabular}

\section{Bajo Ariari}

\begin{tabular}{|c|c|c|c|}
\hline Puerto Concordia & 21131 & 0 & No tiene \\
\hline Mapiripán & 18985 & 0 & No tiene \\
\hline Puerto Rico & 18943 & 1 & Radio (FP) \\
\hline Puerto Lleras & 9372 & 2 & Radio \\
\hline TOTAL MEDIOS REGION DEL ARIARI & & 14 & \\
\hline
\end{tabular}

Fuente: Elaboración propia a partir del mapeo de la FLIP, 2015.

Se estudiaron ocho (8) medios comunitarios del Alto y Medio Ariari, que operan en los municipios de San Juan de Arama, Granada y Cubarral. Se excluyeron de este estudio los medios que operan en el Bajo Ariari.

En un tercer momento, se realizaron grupos focales para documentar la experiencia de cada medio, atendiendo los protocolos formulados por CIMAS (2009) para este tipo de metodología. Esta fase incluyó el análisis de mapas mentales que fueron elaborados por parte de los actores vinculados a los medios comunitarios, para identificar los elementos que, desde su perspectiva, deberían vincularse a un modelo de comunicación comunitaria para la paz.

La información se analizó en atención a lo planteado por González (2013), para quien, desde un punto de vista hermenéutico, la investigación cualitativa aborda lo real desde un punto de vista subjetivo. Esto implica interpretar las vivencias, experiencias, sentires y acciones humanas para explicar los fenómenos sociales (ver figura 2). 
FIGURA 2

Fases de la investigación

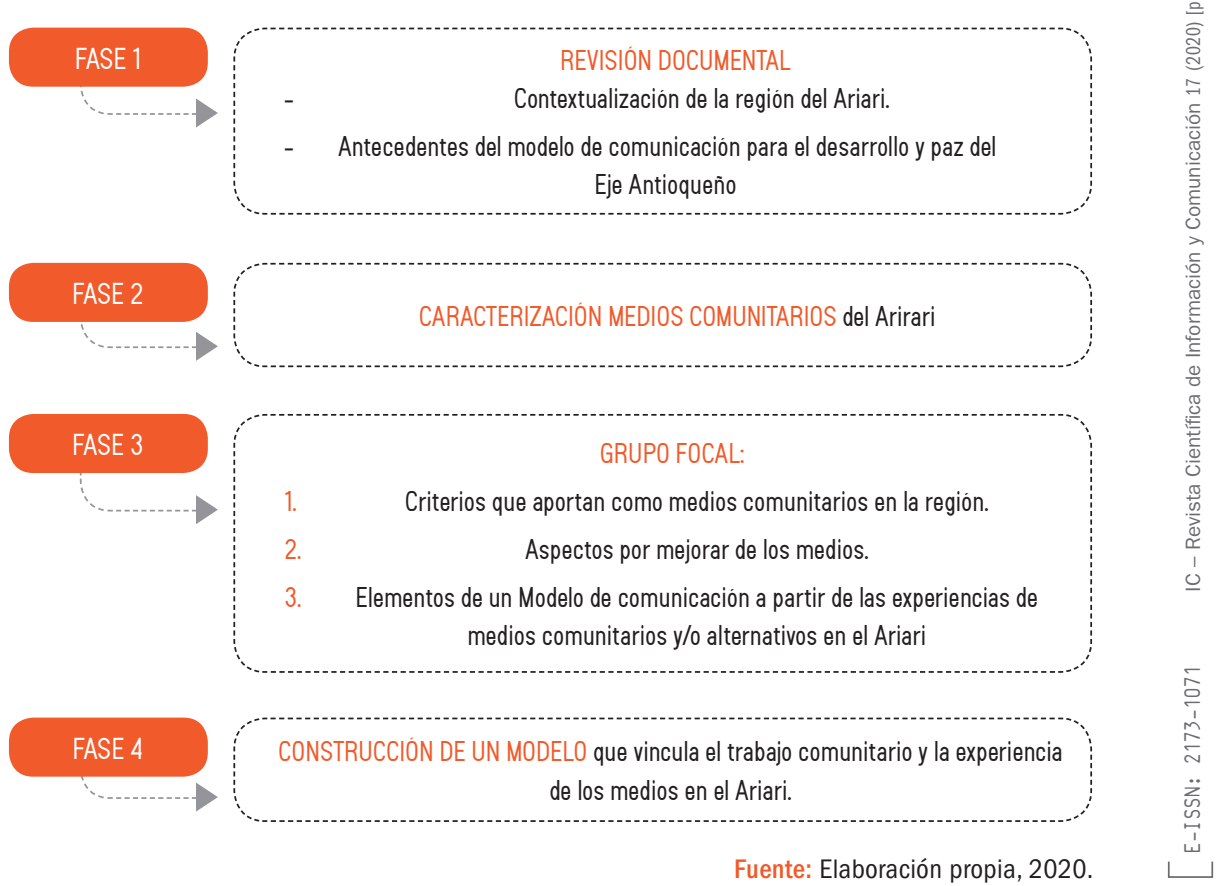

\section{Caracterización de los medios comunitarios del Ariari}

Se consideró identificar los tipos de soporte de los medios comunitarios, así como los segmentos a los que van dirigidos, la periodicidad, sus agendas temáticas, los perfiles del talento humano, los mecanismos de financiación y las oportunidades de mejoramiento.

De los ocho medios de comunicación comunitaria que operan en el Alto y Medio Ariari, cuatro (4) son estaciones de radio, dos (2) son canales de televisión, uno (1) es un sitio web y uno (1) es impreso con circulación mensual. De las 4 estaciones de radio, 3 irradian su señal mediante frecuencia modulada (FM) y al mismo tiempo mediante internet. 
Una de ellas, Me suena FM, emite señal exclusivamente en internet. En cuanto a los dos canales de televisión analizados, emiten en señal abierta y mediante sus plataformas digitales. En términos generales, los medios se soportan principalmente en el tipo de soportes, formatos y géneros propios de su naturaleza, pero todos cuentan con espacios en plataformas digitales para la promoción y difusión de contenidos.

TABLA 2

Medios de comunicación comunitarios y/o alternativos en el Ariari

\begin{tabular}{|c|c|c|c|c|c|}
\hline Medio & Ubicación & Radio & Prensa & TV & Digital \\
\hline Brisas del Tonoa & Cubarral & $x$ & & & $x$ \\
\hline TV Oro Oro & Granada & & & $x$ & $x$ \\
\hline Reporteros de los Hechos & Granada & & $x$ & & $x$ \\
\hline Me suena FM & Granada & $x$ & & & $x$ \\
\hline Camaxagua & $\begin{array}{c}\text { San Juan de } \\
\text { Arama }\end{array}$ & $\mathrm{x}$ & & & $x$ \\
\hline Ondas Don Bosco & Granada & $x$ & & & $x$ \\
\hline Tv Orinoquia & Granada & & & $x$ & $x$ \\
\hline Revista Oro Oro & Granada & & $x$ & & $x$ \\
\hline
\end{tabular}

Fuente: Elaboración propia a partir de las encuestas a medios comunitarios, 2020.

Los medios comunitarios del Ariari difunden sus contenidos de manera general, sin una segmentación de sus audiencias. Las agendas se definen de manera arbitraria y se dirigen al "público en general". Ninguno de los ocho medios de comunicación analizados tiene en su estructura organizativa una unidad destinada a identificar audiencias ni, en consecuencia, a diferenciar espacios ni contenidos que se dirijan a públicos perfilados.

Emisora Ondas Don Bosco es el medio comunitario más antiguo de la región, con 35 años de funcionamiento. Los siete restantes comenzaron a operar en los últimos 11 años (ver tabla 3). 
TABLA 3

Antigüedad de los medios de comunicación comunitarios en el Ariari

\begin{tabular}{|c|c|c|c|c|c|}
\hline Tiempo / Medio & 1- 5 años & 6-10 años & 11-15 años & $15-20$ años & Más de 30 años \\
\hline Periódico Reporteros de los Hechos & & & $x$ & & \\
\hline Revista Oro Oro & & $x$ & & & \\
\hline Emisora Camaxagua & & $x$ & & & \\
\hline Emisora Brisas del Tonoa & & & $x$ & & \\
\hline Emisora Ondas Don Bosco & & & & & $x$ \\
\hline TV Oro Oro & & $x$ & & & \\
\hline TV Orinoquia & & $x$ & & & \\
\hline Radio Me Suena FM & $x$ & & & & \\
\hline
\end{tabular}

Fuente: Elaboración propia a partir de las encuestas a medios comunitarios, 2020.

En cuanto a los tipos de contenido que difunden, los ocho medios presentan agendas variopintas que incluyen desde temas de cultura referidos a las expresiones artísticas y folclóricas del Ariari hasta información periodística relacionada con desarrollos políticos, económicos y deportivos. Aun así, se pudieron identificar algunas preferencias a la hora de tratar determinados campos temáticos. Llama la atención la inclusión de temas religiosos ligados a las prácticas de las iglesias católica y cristianas con presencia en la zona, pese a que las regulaciones legales les impiden a estos medios ofrecer contenidos de carácter ideológico o doctrinal (ver tabla 4). 


\section{TABLA 4 \\ Abordajes temáticos de los medios de comunicación comunitarios en el Ariari}

\begin{tabular}{|c|c|c|c|c|c|c|c|c|c|c|c|c|}
\hline Tema & $\begin{array}{l}\overline{\bar{\pi}} \\
\bar{\delta} \\
\text { œ }\end{array}$ & $\begin{array}{l}\text { 을 } \\
\text { 을 } \\
\text { 岜 }\end{array}$ & 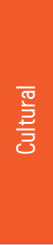 & 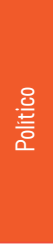 & $\begin{array}{l}\stackrel{8}{ \pm} \\
\frac{0}{0} \\
\stackrel{0}{0}\end{array}$ & $\begin{array}{l}\frac{\pi}{0} \\
\frac{.0}{0} \\
\frac{0}{0} \\
2\end{array}$ & 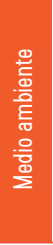 & 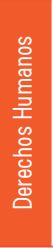 & $\begin{array}{l}\frac{\mathscr{J}}{2} \\
\frac{\mathscr{g}}{2}\end{array}$ & 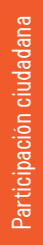 & 岢 & $\begin{array}{l}\dddot{\mathscr{J}} \\
\frac{\text { 응 }}{\bar{\Phi}}\end{array}$ \\
\hline Emisora Brisas del Tonoa & $x$ & & $x$ & & & $x$ & $x$ & & $x$ & $x$ & & \\
\hline TV Oro Oro & $x$ & & $x$ & $x$ & & $x$ & & & $x$ & $x$ & & \\
\hline eriódico Reporteros de los Hechos & $x$ & & $x$ & & & $x$ & & & $x$ & $x$ & & \\
\hline Radio Me Suena FM & $x$ & & $x$ & $x$ & $x$ & $x$ & $x$ & $x$ & $x$ & $x$ & & \\
\hline Emisora Camaxagua & $x$ & & $x$ & & $x$ & $x$ & $x$ & & $x$ & $x$ & & \\
\hline Emisora Ondas Don Bosco & $x$ & & $x$ & & & $x$ & & $x$ & $x$ & $x$ & $x$ & $x$ \\
\hline TV Orinoquia & $x$ & & $x$ & $x$ & & $x$ & & & $x$ & $x$ & & \\
\hline Revista Oro Oro & $x$ & $x$ & $x$ & $x$ & & $x$ & & & $x$ & $x$ & & \\
\hline
\end{tabular}

Fuente: Elaboración propia a partir de las encuestas a medios comunitarios, 2020.

Los equipos de trabajo en estos medios están conformados en un $80 \%$ por personas autóctonas de la región, de las cuales el 85\% son comunicadores empíricos que no han recibido formación académica, pero que desde la práctica han desarrollado habilidades para la producción audiovisual, escritural y radial. Desempeñan funciones que van desde el cubrimiento de eventos noticiosos hasta la producción de formatos. El $20 \%$ de comunicadores foráneos han hecho carrera profesional en comunicación y provienen especialmente de las ciudades de Bogotá, Ibagué y Villavicencio, esta última capital del departamento del Meta.

La mitad de los medios analizados cuenta, en promedio, con equipos de cinco comunicadores, en tanto que tres (3) de ellos cuentan con equipos de entre 10 y 15 personas. Solo uno (1) tiene a disposición 16 personas (ver tabla 5). 


\section{Plantillas de comunicadores en los medios de comunicación comunitarios y/o alternativos en el Ariari}

\begin{tabular}{|c|c|c|c|c|}
\hline Tiempo / Medio & $1-5$ personas & 6-10 personas & $11-15$ personas & $16-20$ personas \\
\hline Periódico Reporteros de los Hechos & $x$ & & & \\
\hline Revista Oro Oro & $x$ & & & \\
\hline Emisora Camaxagua & & & $x$ & \\
\hline Emisora Brisas del Tonoa & & & $x$ & \\
\hline Emisora Ondas Don Bosco & & & $x$ & \\
\hline TV Oro Oro & $x$ & & & $x$ \\
\hline TV Orinoquia & & & & \\
\hline Radio Me Suena FM & $x$ & & & \\
\hline
\end{tabular}

Fuente: Elaboración propia a partir de las encuestas a meedios comunitarịos, 2020.

En lo que toca a la financiación de los medios comunitarios en el Ariari, esta se

ve restringida por normas legales, en razón a que no pueden difundir anuncios comerciales que provengan de campañas ni movimientos políticos, así como tampoco de comunidades religiosas o que representen corrientes ideológicas.

Algunos medios reciben recursos de cooperación internacional y donaciones de fundaciones y organizaciones no gubernamentales. Además de la pauta publicitaria, reciben recursos de campañas cívicas y eventos artísticos y culturales. De manera eventual, los medios comunitarios reciben aportes económicos en compensación por el apoyo a actividades gubernamentales, especialmente de la gobernación del departamento del Meta.

\section{Resultados: factores determinantes de un modelo de comunicación comunitaria}

La lectura de los hallazgos que surgen luego de la aplicación de los instrumentos de investigación permite identificar dos dimensiones en torno a la actuación de los medios comunitarios frente un deseable desarrollo regional: (1) Aportes al desarrollo y (2) Oportunidades de mejora.

En cuanto a los Aportes al desarrollo, se evidencian los espacios de participación ciudadana en los procesos de producción de contenidos y la difusión de información 
asociada al acontecer cercano del Llano. En torno a esto último, los contenidos giran alrededor de temas de medio ambiente, cultura, deporte, agro, contexto social, económico y emprendimiento. Se perciben intenciones hacia la preservación de la memoria y la cultura mediante la exaltación de las tradiciones folclóricas del Llano.

En otro ámbito de actuación, los medios comunitarios abren espacios para el desarrollo de campañas que promueven el cuidado al medio ambiente y la defensa de los Derechos Humanos.

En lo que toca a los aspectos por mejorar, se observa la necesidad de cualificar las plantillas de comunicadores en aspectos como el periodismo digital, la redacción, los procesos editoriales y el manejo de tecnologías de la información y comunicación. Existen sensibles vacíos en materia administrativa que impiden la consolidación de las estructuras organizativas de los medios especialmente en los aspectos técnicos, de calidad y de mejoramiento del recurso humano.

De igual manera, los medios carecen de mecanismos que permitan la identificación de sus audiencias y de estrategias a corto, mediano y largo plazo que viabilicen su crecimiento institucional y potencien su impacto en las comunidades.

En el ámbito externo, los medios comunitarios operan en desventaja frente a los medios de comunicación comercial, lo cual evidencia la necesidad de insistir en reformas a los marcos legales y jurídicos que regulan su funcionamiento. Esto va en coherencia con una mirada desdeñosa por parte del Estado, que no prioriza a los medios comunitarios como espacios para la promoción de la política pública.

En el contexto del periodo de pos-acuerdo, surge como una necesidad la promoción de un "lenguaje para la paz" que desaborde las cargas semánticas asociadas a la guerra y privilegie mensajes enfocados hacia la reconciliación, la inclusión, el perdón y el reconocimiento de las víctimas y victimarios como actores parte de un proceso de restauración social.

\section{Discusión y conclusiones: Modelo de comunicación comunitaria}

En atención a lo planteado por Toro (2014), un modelo de comunicación para el Ariari debería orientarse hacia el Desarrollo Humano Integral y Sostenible. Un escenario deseable de paz no solo comprende la ausencia de guerra, sino la puesta en marcha de un proceso que, según la autora, debe orientarse hacia el desarrollo humano (justo, sustentable 
y perdurable) que se traduzca en la satisfacción de las necesidades básicas y necesarias para que la gente pueda desarrollar toda su potencialidad en busca del bien común.

En esta propuesta se mantienen los ejes centrales del modelo planteado por Toro (2014), pero se vinculan elementos que se derivan de las realidades y contextos propios del Ariari. El modelo se soporta en la operación de los medios comunitarios estudiados, pero con pasibilidades de incluir otros medios existentes o por desarrollarse en la región.

Los medios de comunicación, en este caso los de carácter comunitario, se constituyen en actores esenciales del diálogo público, porque tienen la capacidad de poner en la agenda ciudadana significados, creencias y valores, en suma, representaciones sociales que influyen en la manera de pensar y actuar de sus audiencias. Para Hernández (2004), los medios no solo ponen en circulación contenidos que permiten elaborar representaciones sociales, sino que las expresan para atender a las demandas de los públicos.

El modelo aquí propuesto se enfoca en las tres dimensiones que determinan el funcionamiento, el rol y el impacto de los medios en un entorno social: los enunciadores, el mensaje y las audiencias. El modelo se plantea en términos de encausar acciones y contenidos que contribuyan a la reconfiguración de la realidad social en un escenario deseable de reconciliación, perdón, convivencia y reconstrucción del tejido social.

En este orden de ideas, se parte de pensar los medios comunitarios en tanto provocadores del diálogo público, el modelo en tanto hoja de ruta hacia el cumplimiento de unos objetivos enmarcados en el desarrollo humano sostenible y las audiencias en tanto protagonistas de su propio destino social.

\subsection{Ejes del modelo de comunicación comunitario}

EJE 1 ENUNCIADORES: este primer eje vincula al medio en su dimensión institucional, a los comunicadores responsables de los contenidos y a las fuentes de información que, desde sus propias perspectivas de mundo, influyen en la definición de las agendas. En la dimensión de los enunciadores, el modelo plantea las siguientes acciones:

Fuentes de información: los medios son canales de expresión a las diversas "voces" de la sociedad para enriquecer el debate ciudadano. Esto no tiene que ver solamente con consultar las fuentes y citarlas, como mandan los cánones del periodismo, sino con abrir espacios en los medios 


\section{FIGURA 3 \\ Modelo de Comunicación en un contexto de Pos-acuerdo}

\section{DESARROLLO HUMANO SOSTENIBLE}

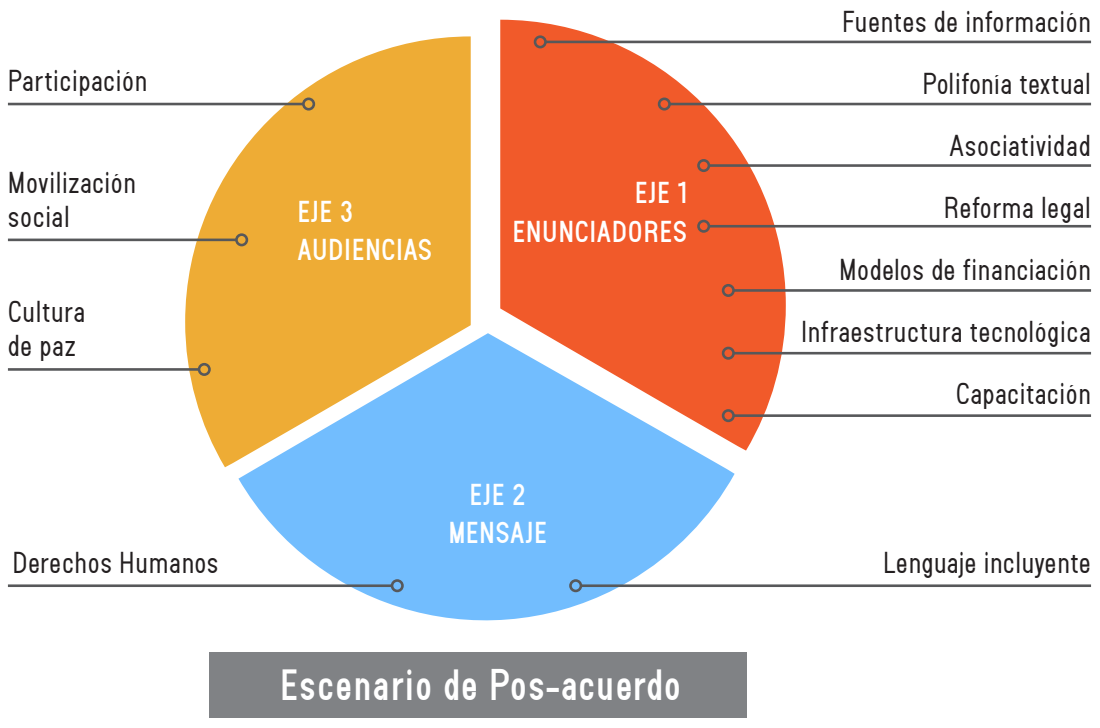

Fuente: Elaboración propia, 2020.

comunitarios en los que esas variadas "voces" pongan en la esfera pública sus particulares formas de ver el mundo. Esto implica vincular información proveniente de organizaciones de la sociedad civil, de instituciones del Estado y, de manera preferencial, de actores del conflicto, esto es: víctimas y victimarios.

- Polifonía textual: la producción de los textos es producto de diversos enunciadores. El periodismo, como lo ha afirmado Moyano (2007), se vale de diferentes formas de reproducir la palabra ajena, no solo para referirla, sino para "caracterizarla e interpretarla". Pese a esa diversidad de enunciadores, el comunicador consolida, al final, un texto cuyo responsable global es él y el medio al que representa (Ducrot, 1984, pág. 
258). Le compete a los medios comunitarios la construcción de discursos que conjuguen diferentes posturas ideológicas en aras de aportar a construcción de consensos, pero también de disensos.

Asociatividad: la asociatividad es un término que aplica para relaciones duraderas que buscan la interacción grupal, compartiendo ideales formal o casualmente. Exigen de un consenso, así como de metas y valores compartidos. En este sentido, se sugiere que los medios trabajen mancomunadamente, no solo para dar un contexto de la realidad de forma más o menos objetiva, sino para potenciar sus cubrimientos e impactos en las audiencias.

No se habla aquí exclusivamente de asociaciones entre medios, sino con otro tipo de instituciones, públicas y privados, en aras de conseguir objetivos comunes. Esto permitirá considerar estrategias que les permitas a los medios comunitarios conquistar espacios tradicionalmente reservados a otros medios con cubrimiento en la región.

- Reforma legal: el modelo planteado aquí vincula procesos de integración encaminados a procesos de debate y presión pública para reformar los marcos legales y jurídicos que regulan la existencia y operación de Ios medios comunitarios. Estos procesos deben promoverse desde los ámbitos municipales y trascenderlos hacia lo nacional por la vía de las agremiaciones ya existentes en este ámbito.

Modelos de financiación: los medios deben contemplar mecanismos y estrategias que aseguren su estabilidad financiera. El modelo planta la estructuración de portafolios de servicios que les permitan a los medios formas de financiación, estrategias comerciales, de mercadeo y publicidad, y diversificación en su oferta de servicio.

Infraestructura tecnológica: Las nuevas formas de presentar la información exigen tecnologías de software y hardware que puedan dar soporte a lo audiovisual, escritural y sonoro, extendiendo no solo en cobertura, sino haciendo presencia en formatos que permitan la interacción con las audiencias. 
- Capacitación: el modelo sugiere planes permanentes de capacitación de sus plantillas de personal que suplan los vacíos de formación profesional, especialmente en materia técnica y conceptual.

EJE 2 MENSAJE: el escenario de pos-acuerdo en la región del Ariari, de cara a la construcción de un escenario de reconciliación y convivencia ciudadana y en la búsqueda de un desarrollo humano sostenible, demanda un replanteamiento en la manera como se presentan los contenidos que sirvan de insumo para el diálogo público. En este sentido, el modelo planea los siguientes aspectos:

- Lenguaje incluyente: el modelo plantea que los medios comunitarios adopten un lenguaje de paz, incluyente, que reconozca al "otro", que no excluya y que promueva el entendimiento en la diferencia. En palabras de Tíjaro (2015, pág. 2), “los desafíos de la deconstrucción del lenguaje hegemónico en la cultura son substanciales, por lo menos en términos del uso del lenguaje como detonador de brechas y generador de más diferencias al no reconocer al interlocutor como válido por diversas razones, principalmente diferenciales".

- Derechos Humanos: el modelo plantea priorizar contenidos relacionados con la promoción de los derechos humanos, en el convencimiento de que estos son pilar de todo proceso que propenda por una cultura de paz. Igualmente, reafirmar la tendencia a privilegiar los temas de participación ciudadana como condición fundamental en la construcción de políticas públicas de beneficio comunitario.

EJE 3 AUDIENCIAS: el modelo contempla la vinculación de las audiencias en la definición de los temas de agenda y en la generación de un diálogo interactivo, por ejemplo, por la vía de los comités editoriales o mediante la apertura de canales de diálogo en las redes sociales. De esta manera, se sugiere una "coresponsabilidad" de las audiencias en la definición de las agendas. En este eje, el modelo contempla los siguientes aspectos: 
Participación y movilización social: En cuanto a la promoción de la participación ciudadana, el modelo se apoya en lo afirmado por la Unesco acerca de la naturaleza de los medios comunitarios. En esencia, esta organización ha señalado:

Los medios comunitarios demuestran una actitud localista y una tendencia a la libre circulación de las ideas y opiniones. Se proponen educar y entretener, informar y divertir, y crear un vasto espacio en el cual los auditores puedan participar y confrontarse con los demás, así como con los dirigentes políticos. Estas iniciativas tienden a ser pequeñas, basadas en la comunidad y administradas por ella, contando con un apoyo local que puede comprender publicidad pero que más a menudo consiste en donaciones y trabajo voluntario (UNESC0, 2008, pág. 1).

En este sentido, el modelo planea la necesidad de que los medios comunitarios no solo promuevan hacia afuera la participación de los diferentes actores sociales, sino que los vinculen en los procesos de definición de las agendas, de las estructuras organizacionales y de los mecanismos de intervención en lo público.

En lo que toca a la movilización social, el modelo acoge los planteamientos de Unicef (s/f) en el sentido de que los medios comunitarios pueden servir al propósito de elevar los niveles de conciencia y conquista de objetivos comunes mediante el diálogo y posibilitar "el cambio por medio del accionar de diversos protagonistas de esfuerzos interrelacionados y complementarios" Pág. 1.

- Cultura de paz: La paz exige un proceso dinámico y participativo que promueva el diálogo y solucione los conflictos a partir del entendimiento y la cooperación mutua (Naciones Unidas, 1999). Una cultura de paz vincula valores y comportamientos que aseguran el respeto a la vida, a la integridad territorial, a la independencia y a los Derechos Humanos. En la Declaración sobre una Cultura de Paz (1999) de las Naciones Unidas se establece el papel fundamental y educativo que los medios 
de comunicación le aportan a una cultura de paz, desempeñando una función clave en la promoción de estos valores. En este orden de ideas, el modelo aquí propuesto vincula la promoción de una cultura de paz como uno de los propósitos de los medios comunitarios en la búsqueda del desarrollo humano sostenible en esa región de Colombia.

Por último, el modelo hace referencia a la necesidad de una "conversación" permanente entre los diferentes actores sociales en torno a la naturaleza y los objetivos de los medios comunitarios ante el reto de transformar la sociedad del Ariari y conseguir ese desarrollo humano sostenible deseable.

\section{Bibliografía}

ADR y FAO. (2019). https://www.adr.gov.co. (A. d. agricultura, Ed.) Recuperado de https://www.adr.gov.co/servicios/pidaret/META\%20TOM0\%201.pdf [Consultado el 03-10-2019]

Ahlert, A. (2007). Ética y Derechos Humanos: principios educacionales para una sociedad democrática. Polis, revista Latinoamericana(16), 1-13.

Alzate. M y Dono, M. (2017). Reconciliación social como estrategia para la transformación de los conflictos sociopolíticos, variables asociadas e instrumentos de medición. Universitas Psycilogica, 16(3).

Caracheo, F. (2002). Modelo educativo (propuesta de diseño), (Dirección General de Institutos Tecnológicos ed.). México: CIDET.

Castillo, C. M. (septiembre- diciembre de 2017). El papel de la participación ciudadana en las políticas públicas, bajo el actual escenario de la gobernanza: reflexiones teóricas. Revista CS (23), 157,180.

Cerda, H. (2011). Los elementos de la investigación cómo reconocerlos, diseñarlos y construirlos. Bogotá: Editorial Magisterio.

Chacón, J. C. (2016). Características de los medios comunitarios y / o alternativos de Bogotá (Colombia). (F. d.-U. Facultad de Comunicación Audiovisual - Politécnico 
Colombiano Jaime Isaza Cadavid, Ed.) Revista Luciérnaga(18), 63-83.

Chneider, S. P. (2006). Territorio y enfoque territorial: de las referencias cognitivas a los aporte aplicados al análisis de los procesos sociales rurales. Buenos Aires: Ed. Ciccus.

CIMAS, O. I. (2009). Metodología participativas . 91.

Cortina, A. (2014). Conferencia ¿Qué es y para qué siver la ética?

Duran S. y Parra, M. (Marzo de 2018). Diversidad cultural y fomento de valores en la convivencia ciudadana. Estrategias gerenciales para promover el cambio organizacional en MIPYMES, 1-21.

Ducrot, O. (1984). El Decir y lo dicho. (S. Vasallo, Trad.) Buenos Aires: Hachette.

Elorrieta, B. (2019). https://www.unibarcelona.com/. (UNIBA, Editor, \& C. U. Barcelona: Productor) Recuperado de https://www.unibarcelona.com/int/ actualidad/noticias/como-medir-la-sostenibilidad-en-la-planificacion-territorial

Fernández, L. y., \& Gutiérrez, M. (2013). Bienestar Social, Económico y Ambiental para las Presentes y Futuras Generaciones. Bienestar Social, Económ, 24(2), 121,130 .

FIP, USAID, \& OIM. (2013). http://www.ideaspaz.org/. Recuperado el 20 de septiembre de 2019, de Dinámica del conflicto armado en Meta y su impacto humanitario: $\quad$ http://archive.ideaspaz.org/images/DocumentoMonitoreo_ ConflictoArmado_Meta_Agosto\%20Final\%202013-correcciones\%20ELI\%20.pdf

Galtung, J. (1996). Peace by peaceful means: Peace and Conflict, Development and Civilization. London: Sage Publications.

Gonzales, E. (2013). Acerca del estado de la cuestión o sobre un pasado reciente en la investigación cualitativa con enfoque hermenéutico. Unipluriversidad,. 13(1), 60-63.

Hernández Vivas, D. I. (21 de Julio de 2004). Representaciones sociales y medios de comunicación: una relación complementaria. (C. Schumacher, Ed.) Reportes 
Escuela de Ciencias Humanas(54), 1-11.

Instituto Departamental de Cultura del Meta. (2016). http://www.culturameta.gov. co. Recuperado de http://www.culturameta.gov.co/content/departamento-meta [Consultado el 02-08-2019]

Islas, A., \& Vera-Hernánez D \& Miranda-Medina, C. ( 2018). La cultura de paz en las políticas de educación superior de México, Colombia y El Salvador. Revista Educación y Humanismo, 20(34), 312-325.

Martín-Barbero, J. (2010). Convergencia digital y diversidad cultural. En D. De Moraes (Comp.), Mutaciones de lo visible. Buenos Aires: Paidós.

Martínez Valle, L. (enero-marzo de 2012). Apuntes para pensar el territorio desde una dimensión social . (U. d. Sinos, Ed.) Ciências Sociais Unisinos, 48(1), 12-18.

Mendoza, Z. G. (2008). https://www.ciasporlapaz.com. (C. d. Social, Productor, \& Ciudad de México) Recuperado de https://www.ciasporlapaz. com/app/download/7631636756/El\%20Tejido\%20Social-DEFINICION. pdf?t=1520870161. [Consultado el 10-09-2019]

Moyano, S. I. (2007). El Discurso narrativizado y las normalizaciones citativas: dos formas solapadas de caracterizar la palabra ajena. Recuperado de http://www. memoria.fahce.unlp.edu.ar/tesis/te.227/te.227.pdf [Consultado el 09-04-2013]

Naciones Unidas. (1999). Declaración y Programa de Acción sobre una Cultura de Paz. Quincuagésimo tercer período de sesiones.

Pino, M. J. (Julio - Diciembre de 2014). Relación entre el concepto de verdad y el concepto de interpetación. Revista Fundación Universitaria Luis Amigó (histórico), 1(2), 97-102.

PNUD. (2015). El departamento del Meta Frente a los Objetivos de Desarrollo del Milenio. Recuperado de https://www.cepal.org/: https://www.cepal.org/MDG/ noticias/paginas/6/44336/Meta_final.pdf [Consultado el 12-10-2019]

(2015). https://www.undp.org. (P. d. Desarrollo, Productor) Recuperado el 20 de Octubre de 2019, de Objetivos de Desarrollo Sostenible: https://www. undp.org/content/undp/es/home/sustainable-development-goals.html

PNUD y OEA. (2013). Guía práctica del Diálgo Democrático. Recuperado el 8 de Agosto de 2019, de https://www.oas.org: https://www.oas.org/es/sap/dsdme/ 
pubs/guia_s.pdf

Prieto. (2009). Rumbo a Ítaca: la participación ciudadana municipal en los albores del siglo XXI. . XXII Concurso del CLAD sobre Reforma del Estado y Modernización de la Administración Pública, Caracas .

Reguero, N. (19 de junio de 2008). http://www.processocom.org. Recuperado el 3 de agosto de 2019, de Jesús Martín-Barbero propone nuevas formas de investigar la comunicación y la cultura: http://www.processocom.org/2008/06/19/jesusmartin-barbero-propone-nuevas-formas-de-investigar-la-comunicacion-y-la-cultura/

Rueda, M. (2007). La investigación etnográfica y/ o cualitativa y la enseñanza en la universidad. Revista Mexicana de investigación educativa, 12(34), 1021 - 1041.

Sampieri, Baptista, \& Collado. (2010). Metodología de la investigación. México D.F: Mc. Graw Hill.

Sánchez, V. A. (1 de Agosto de 2015). Nuevos' valores en la práctica psicosocial y comunitaria: Autonomía compartida, auto-cuidado, desarrollo humano, empoderamiento y justicia social. Universitas Psychologica, 4(14), 1235-1244.

Sesento, L. (Septiembre de 2008). Modelo sistémico basado en competencias para instituciones educativas públicas. Morelia, Michoacán: Centro de investigación y desarrollo del Estado de Michoacán. Recuperado de http://www.eumed.net/tesisdoctorales/2012/lsg/concepto_modelo.html

Sztompka, P. (1995). Sociología del cambio social. Madrid: Alianza Editorial.

Tíjaro, A. (Julio de 2015). Comunicación para la paz. Interacción Revista de comunicación Educativa CEDAL(58).

Valencia Lopez, E. (2010). Revisión Documental en el proceso de Investigación. (U. T. Pereira, Ed.) 5. Obtenido de Re: https://univirtual.utp.edu.co/pandora/ recursos/1000/1771/1771.pdf

UNICEF. (s/f). Movilización social. Recuperado el 15 de Diciembre de 2019, de https://www.unicef.org: https://www.unicef.org/spanish/cbsc/index_42347.html

Velézquez, \& Vargas - Hernádez, J. (enero - diciembre de 2012). La sustentabilidad como modelo de desarrolol responsable y competitivo. (Facultad de Ingeniería de 
Recursos Naturales y del Ambiente, Ed.) (11), 97-107.

Villanueva, S., \& Barranquero \& Ramos, M. (2016). Los medios comunitarios, libres y ciudadanos en el Estado español.Territorios, tecnologías y valore. Revista de estudios culturales de la Universitat Jaume I, XV, 99-118.

Zúñiga, D., \& Grattan, J. S. (2017). Papel de las radios comunitarias en el proceso de consolidación de la paz en Colombia. En Humanidades digitales, diálogo de saberes y prácticas colaborativas en red - Cátedra Unesco de comunicación (pág. 262). Bogotá: Pontificia Universidad Javeriana. Obtenido de Papel de las radios comunitarias en el proceso de consolidación de la paz en Colombia: https://www. javeriana.edu.co/unesco/humanidadesDigitales/ponencias/IV_24.html 